\title{
Developing a model for cystic fibrosis sociomicrobiology based on antibiotic and environmental stress
}

\author{
Susana Patrícia Lopes ${ }^{\mathrm{a}, *}$, Nuno Filipe Azevedo ${ }^{\mathrm{b}}$, Maria Olívia Pereira ${ }^{\mathrm{a}}$ \\ a Centre of Biological Engineering, LIBRO - Laboratório de Investigação em Biofilmes Rosário Oliveira, University of Minho, Campus de Gualtar, 4710 -057 Braga, Portugal \\ b LEPABE - Dep. of Chemical Engineering, Faculty of Engineering, University of Porto, Rua Dr. Roberto Frias, s/n, $4200-465$ Porto, Portugal
}

\section{A R T I C L E I N F O}

\section{Keywords:}

Antibiotic therapy

Cystic fibrosis

Interspecies interactions

Polymicrobial biofilms

Pseudomonas aeruginosa

\begin{abstract}
A B S T R A C T
Cystic fibrosis (CF) infections are invariably biofilm-mediated and polymicrobial, being safe to assume that a myriad of factors affects the sociomicrobiology within the $\mathrm{CF}$ infection site and modulate the CF community dynamics, by shaping their social activities, overall functions, virulence, ultimately affecting disease outcome. This work aimed to assess changes in the dynamics (particularly on the microbial composition) of dual-/threespecies biofilms involving CF-classical (Pseudomonas aeruginosa) and unusual species (Inquilinus limosus and Dolosigranulum pigrum), according to variable oxygen conditions and antibiotic exposure.

Low fluctuations in biofilm compositions were observed across distinct oxygen environments, with dualspecies biofilms exhibiting similar relative proportions and $P$. aeruginosa and/or $D$. pigrum populations dominating three-species consortia. Once exposed to antibiotics, biofilms displayed high resistance profiles, and microbial compositions, distributions, and microbial interactions significantly challenged. The antibiotic/oxygen environment supported such fluctuations, which enhanced for three-species communities.

In conclusion, antibiotic therapy hugely disturbed CF communities' dynamics, inducing significant compositional changes on multispecies consortia. Clearly, multiple perturbations may disturb this dynamic, giving rise to various microbiological scenarios in vivo, and affecting disease phenotype. Therefore, an appreciation of the ecological/evolutionary nature within CF communities will be useful for the optimal use of current therapies and for newer breakthroughs on CF antibiotherapy.
\end{abstract}

\section{Introduction}

According to the World Health Organization, infectious respiratory diseases are a major cause of morbidity and mortality worldwide, accounting for 4.18 million premature deaths per year (WHO, 2013). It is believed that most - if not all - of these infections are associated with biofilms, which represent a critical health-care burden (Bjarnsholt et al., 2013) because of their great contribution to the emergence and dissemination of antibiotic resistance. Cystic fibrosis (CF) is a common heritable genetic disorder (100 000 people estimated to be affected) (Davies et al., 2014) with major debilitation in the respiratory tract, where patients are prone to develop severe biofilm-related infections. $\mathrm{CF}$ infection was originally characterized as monomicrobial, likely due to the extensive use of culture-dependent isolation techniques. However, the advent of affordable high-resolution molecular technologies has dramatically changed our view on the true diversity of microbes present in the CF airways (Lopes et al., 2015). Indeed, the large variety and concentration of microbes includes not only the key pathogens habitually recovered (e.g. Pseudomonas aeruginosa) but also unusual species (e.g. Inquilinus limosus, Stenotrophomonas maltophilia, Dolosigranulum pigrum, Pneumoncystis jirovecii, Scedosporium spp., adenovirus, rhinovirus, etc.) (Bittar et al., 2008; Coenye et al., 2002; Pederiva et al., 2012). Though that CF aetiology is invariably polymicrobial, most research has focused on specific pathogens, failing to consider the role of unusual microbes in the disease progression (Lopes et al., 2014; , 2015; Magalhães et al., 2016; Waters, 2012) or their social interactions with established pathogens (Lopes et al., 2012). Accordingly, I. limosus (a gram-negative aerobe with a very mucoid phenotype) (Coenye et al., 2002) and D. pigrum (a gram-positive, facultative anaerobe, arranged in pairs, tetrads, and clusters) (Aguirre et al., 1993) are less typical bacteria reported in expectorated CF patients (Bittar et al., 2008). Whilst the pathogenic potential of $I$. limosus has been already pointed (Chiron et al., 2005), information on D. pigrum is still limited and its role in CF infection unclear. The upper respiratory tract is thought to be its natural habitat (Laclaire and Facklam, 2000), but its occurrence in other polymicrobial contexts (Hoedemaekers et al., 2006; Lecuyer et al., 2007) has raise up interest in evaluating its pathogenic potential in CF polymicrobial communities. Interestingly, earlier in vitro studies have

\footnotetext{
* Corresponding author.

E-mail address: supat@deb.uminho.pt (S.P. Lopes).
} 
unravelling the contribute of $I$. limosus and D. pigrum for the resilience of CF-associated biofilms to antibiotic treatment (Lopes et al., 2014; Lopes et al., 2012), thereby allowing to hypothesize their influence in the behaviour of coexisting species or even in benefiting the whole community. It is likely that the multifaceted CF microbiome promotes for species-specific relationships, often necessary for the development and structure of the community and other regulatory activities that coordinate the whole community (Magalhães et al., 2016; TolkerNielsen and Molin, 2000). In addition, it is now evident that microbial communities can be modulated by a wide variety of other factors that, altogether, give rise to various microbiological scenarios seen in vivo and are likely to affect disease phenotype and the clinical outcome (Guss et al., 2011; Magalhães et al., 2016; Peters et al., 2012a; Stressmann et al., 2011), as has been determined for other pathologies (Azevedo et al., 2014; Azevedo et al., 2016; LiPuma, 2014; Peters et al., 2012b). For instance, the physicochemical characteristics of the airways; the administration of the antibiotic therapy, (maintenance vs. treatment of episodes of acute exacerbation); the nature of the pre-existing microbial community; the concomitant airway inflammation resulting from chronic infections as well as patient- and/or disease-associated issues (increasing age, disease progression, reduced lung function) have been suggested to have a crucial impact on the dynamics of CF polymicrobial communities (Cuthbertson et al., 2016; KlepacCeraj et al., 2010; Rogers et al., 2015; Serisier, 2013; van der Gast et al., 2014; Willner et al., 2012; Zhao et al., 2012). Altogether, these factors may drive to social, structural and functional diversification and consequently impact the ecology and evolution in natural species populations (Allen and Banfield, 2005; Schloter et al., 2000). Although it is still almost impossible to predict biofilm behavior and the dynamics of a community, it is now increasingly recognized that its regulatory networks (e.g. social activities, overall functions, virulence traits, etc.) are often strictly evolved in response to those highly variable stimuli (Allen and Banfield, 2005; Nadell et al., 2016), and improving and directing efforts to better understand how polymicrobial infections behave and respond to surrounding stresses may give insights to better predict disease progression and to define more effective antimicrobial strategies.

In this scope, this study aimed at deeply investigating the behavior of I. limosus and D. pigrum when associated with the CF-conventional pathogen $P$. aeruginosa in dual-species and even in three-species populations. The response of these multispecies communities to aerobic (AER) microaerophilic (MAER) and anaerobic (ANAER) conditions resembling the $\mathrm{CF}$ airways and to subsequent antibiotic interventions was further examined in detail. Thus, changes in the biofilm-formation ability, antibiotic resistance profiles, bacterial killings, microbial composition and distribution within the multispecies biofilms were assessed.

\section{Material and methods}

\subsection{Bacterial strains and culture conditions}

The bacterial strains used in this work were: $P$. aeruginosa (strain UCBPP- PA14), I. limosus (strain M53, isolated from CF sputum), and D. pigrum (CIP 104051T, purchased from Institute Pasteur Collection, Paris, France). All strains were stored at $-70{ }^{\circ} \mathrm{C}$ in $20 \%$ (v/v) glycerol. Prior to inoculation, bacteria were subcultured twice from the frozen stock preparations onto tryptic soy broth (TSB, Liofilchem, Italy) supplemented with $1.2 \%(\mathrm{w} / \mathrm{v})$ agar (TSA, Liofilchem) plates and incubated at $37^{\circ} \mathrm{C}$ for $24-48 \mathrm{~h}$.

\subsection{Formation of multispecies biofilms under variable oxygen conditions}

The formation of dual- and three-species biofilms formed by $P$. aeruginosa, I. limosus and D. pigrum under AER, MAER and ANAER conditions was performed as described elsewhere (Lopes et al., 2014).
Briefly, for preparing biofilm inocula, several colonies from a fresh subculture of each strain were suspended in TSB to match a 1.0 McFarland standard. This was further diluted in broth medium (TSB) to obtain pure cultures with $\sim 10^{7}$ cells $/ \mathrm{mL}$. For dual-species cultures, the suspended inoculum of each species was combined in a 1:1 ratio, whereas the three-species cultures were obtained by mixing the three pure bacterial suspensions in equal proportions. Bacterial suspensions were dispensed in standard 96-well microtiter plate wells and incubated at $37{ }^{\circ} \mathrm{C}$ and $120 \mathrm{rpm}$ until achieving the threshold the cell concentration interval of $2 \times 10^{5}$ to $2 \times 10^{6} \mathrm{CFU} / \mathrm{cm}^{2}$ (determined by previous established biofilm growth curves) (Lopes et al., 2014). To resemble the oxygen environments of $\mathrm{CF}$ airways, plates were incubated at different conditions: AER (n-biotek, Model NB-205Q, Korea incubator), MAER (Thermo Scientific, Forma 311, USA incubator, calibrated with $5 \% \mathrm{v} / \mathrm{v}$ $\mathrm{CO}_{2}$ ) and ANAER (AnaeroGen Atmosphere Generation system, Oxoid, Cambridge, UK). The ANAER atmosphere was created by sealing the plates containing the cells suspensions in plastic boxes with AnaeroGen sachets (Oxoid), which reduces oxygen concentration to below $1 \%(\mathrm{v} /$ v). This oxygen reduction was confirmed by using anaerobic indicator strips (Oxoid) (see Fig. S1 in Supplementary Material).

\subsection{Quantification of biomass and metabolic activity of biofilms}

After biofilm formation, the content of microtiter plate wells (planktonic cell fraction) was discarded, the wells washed once with sterile $0.9 \%(\mathrm{w} / \mathrm{v})$ saline solution to remove non-adherent and weakly adherent bacteria, and biofilms were quantified for biomass and metabolic activity. Biofilm mass quantification was based on staining biofilms with crystal violet (CV), with protocol adapted from Stepanovic et al. (Stepanovic et al., 2000). Briefly, the wells were air dried for $10 \mathrm{~min}$ before fixing attached bacteria with pure methanol (Fisher Scientific, Leicestershire, UK) for $15 \mathrm{~min}$. Then, biofilms were stained for 1 min with 1\% (v/v) CV (Merck, Germany). The excess stain was removed by washing twice the wells with sterile distilled water. Lastly, the bound dye was dissolved in pure methanol $(200 \mu \mathrm{L} /$ well $)$ and the optical density (OD) was read at $550 \mathrm{~nm}\left(\mathrm{OD}_{550 \mathrm{~nm}}\right)$ in a microtiter plate reader (Model Sunrise-basic Tecan, Austria).

The metabolic activity of biofilms was measured using the 2,3-bis (2-methoxy-4-nitro-5-sulfophenyl)-2Htetrazolium-5-carboxanilide sodium salt (XTT) colorimetric method, initially described by Stevens and Olsen (Stevens and Olsen, 1993), with some modifications. Basically, after biofilm growth and washing procedures, $200 \mu \mathrm{L}$ of a combined solution of XTT (Sigma) and phenazine methosulfate (PMS) (Sigma) were added to each well in order to obtain a final concentration of $150 \mathrm{mg} / \mathrm{L}$ of XTT and $10 \mathrm{mg} / \mathrm{L}$ of PMS. After that, plates were incubated at $37^{\circ} \mathrm{C}$ for $3 \mathrm{~h}$ in the dark. Biofilm metabolic activity was determined by reading the $\mathrm{OD}$ at $490 \mathrm{~nm}\left(\mathrm{OD}_{490 \mathrm{~nm}}\right)$ in each well.

\subsection{Determination of the competitive index (CI) and the relative increase ratio (RIR)}

In order to determine whether the species could compete with each other in multispecies biofilms, the Competitive Index (CI) was defined as the $\mathrm{X} / \mathrm{Y}$ ratio within the output sample (immediately after biofilm formation) divided by the corresponding ratio in the inoculum (input):

\section{$\mathrm{CI}[\mathrm{X}$ vs $\mathrm{Y}]=\left[\mathrm{N}(\mathrm{X} / \mathrm{Y})_{\text {output }} / \mathrm{N}(\mathrm{X} / \mathrm{Y})_{\text {input }}\right]$}

where $\mathbf{N}$ is the value of $\mathrm{CFU} / \mathrm{cm}^{2}$ obtained for the species $\mathrm{X}$ or $\mathrm{Y}$ in the biofilm, in the output (at fixed times) and input ( $\mathrm{t}=0$ or the inoculum) sample (Macho et al., 2007). For statistical analyses, CI values were first subjected to a Log transformation for normal distribution, then interpreted as follows: a CI value equal to 0 indicates equal competition of species $\mathrm{X}$ and $\mathrm{Y}$ in the consortium; a positive $\mathrm{CI}$ value indicates a competitive advantage for the species $\mathrm{X}$; a negative $\mathrm{CI}$ value indicates a competitive advantage for the species Y. Similarly, the 
Relative Increase Ratio (RIR) was calculated and based on the growth results obtained from the single-species biofilms formed by each strain. Calculations were adapted from (Macho et al., 2007). Statistical significant differences between CI and RIR means for each case were suggestive of a meaningful competition between the species in the consortia.

\subsection{Biofilm resistance profiles}

Antibiotics susceptibilities of biofilms were determined by adapting the protocol described by Ceri et al. (Ceri et al., 1999). For that, preestablished biofilms (containing $2 \times 10^{5}$ to $2 \times 10^{6} \mathrm{CFU} / \mathrm{cm}^{2}$ ) formed on the microtiter plate wells were exposed to increasing 2 -fold concentrations (ranging from 2 to $1024 \mathrm{mg} / \mathrm{L}$ ) of 9 clinically-relevant antibiotics: tobramycin (TOB), gentamicin (GEN), levofloxacin (LVF), ciprofloxacin (CIP), clindamycin (CLI), cefotaxime (CTX), chloramphenicol (CAM), rifampicin (RIF) and aztreonam (ATM, all from Sigma), prepared in cation-adjusted Mueller-Hinton broth (CAMHB). Plates were then incubated at $37{ }^{\circ} \mathrm{C}$, under AER, MAER and ANAER environments, as described above. Following $24 \mathrm{~h}$ of antibiotic exposure, planktonic cell fractions (supernatant) released for treatedbiofilms were transferred to new microtiter plates and the minimum inhibitory concentrations (MICs) were obtained by reading the OD at $650 \mathrm{~nm}\left(\mathrm{OD}_{650 \mathrm{~nm}}\right)$, where $\mathrm{OD}_{650 \mathrm{~nm}}<0.1$ was evidence of bacterial growth inhibition. Biofilms attached to the wells were rinsed twice with saline solution and disrupted (by sonication with an ultrasound bath) into TSB supplemented with $1 \%(\mathrm{v} / \mathrm{v})$ tween $^{\circ} 20$ (Fisher Scientific, NJ, USA). The minimum biofilm eradication concentration (MBEC), corresponding to the lowest antibiotic concentration able to inhibit biofilm growth, was determined after plating serial dilutions of disrupted biofilms samples onto TSA.

\subsection{Assessment of bacterial killings within biofilms after antibiotic exposure}

The efficacy of the aforementioned antibiotics at concentrations equal to 2, 128 and $1024 \mathrm{mg} / \mathrm{L}$ was evaluated for bacterial killings within biofilms, which was expressed in terms of percentage reduction in biofilm-cell numbers. After biofilms being exposed to antibiotics (as previously described), total cultivable cell numbers were enumerated by plating 10 -fold serially diluted biofilm-detached samples onto TSA plates. Biofilm-cell killing was reported as the percentage of log reduction calculated to the relative cell count at the time of initiation of antibiotic exposure, according to the following:

Biofilm-cell reduction $(\%)=100-[\mathrm{N}$ (after exposure to the antibiotic) $/ \mathrm{N}$ (prior to antibiotic exposure) $\times 100$ ]

where $\mathbf{N}$ is the value of $\mathrm{CFU} / \mathrm{cm}^{2}$ present in the biofilm determined before or after being exposed to the antibiotic at the desired concentration.

\subsection{Determination of biofilms composition}

In this work, the relative species composition within polymicrobial biofilms was estimated before and after antibiotic exposure. Basically, after the biofilms rinse step, the wells were filled with saline solution ( $200 \mu \mathrm{L} /$ well) and biofilms formed in the wells were detached by sonication using an ultrasound bath (Sonicor, model SC-52, UK) operating at $50 \mathrm{kHz}$, during $10 \mathrm{~min}$ and then resuspended by pipetting up and down. The sonication step was previously optimized to ensure that all cells were detached from the wells of the microtiter plate, while avoiding cell disruption (data not shown). The estimation of total biofilms-cells was enumerated by using TSA whereas selective agar media was used to discriminate each bacterial population in the consortia: Pseudomonas isolation agar (PIA; Sigma) and Burkholderia cepacia selective agar, (BCSA; Oxoid Ltd, Hampshire, UK) supplemented with 300
$000 \mathrm{IU} / \mathrm{L}$ polymyxin B (Biochrom, Berlin, Germany) and $100 \mathrm{mg} / \mathrm{L} \mathrm{ti-}$ carcillin (Sigma) for P. aeruginosa and I. limosus isolation, respectively. Due to the lack of a specific selective medium for $D$. pigrum, this species was estimated by the difference between the average total cell number (in TSA) and the average of other bacteria presented in the consortia.

\subsection{Multiplex PNA FISH assay applied to biofilms}

In order to discriminate the different populations within the polymicrobial biofilms and to confirm (qualitatively) the results obtained from culture, fluorescence in situ hybridization (FISH) using peptidenucleic acid (PNA) probes followed by an additional staining step with 4', 6-diamidino-2-phenylindole (DAPI; Sigma) was performed directly on polymicrobial consortia. Firstly, biofilms were formed on the surface of Nunc $^{\mathrm{TM}}$ Lab-Tek $^{\mathrm{TM}}$ II Chamber Slide ${ }^{\mathrm{TM}}$ System (Nalge Nunc International; Naperville, IL, USA). After achieving the threshold concentration, biofilms were washed twice with $1 \mathrm{~mL}$ of sterilized deionized water and air dried at $\sim 60{ }^{\circ} \mathrm{C}$ for $15 \mathrm{~min}$. Biofilms were then fixed with methanol $(100 \% \mathrm{v} / \mathrm{v})$ for $20 \mathrm{~min}$, followed by $4 \%(\mathrm{w} / \mathrm{v})$ paraformaldehyde and $50 \%(\mathrm{v} / \mathrm{v})$ ethanol $(10 \mathrm{~min}$ each), at room temperature. After the fixation step, the surfaces of the chamber slide were covered with $20 \mu \mathrm{L}$ of hybridization solution with a mixture of two probes at $200 \mathrm{nM}$ and covered with coverslips. These PNA probes, designated Paer565 and Ilim569, were previously developed, optimized and validated (Lopes et al., 2017) in order to specifically detect $P$. aeruginosa and I. limosus within polymicrobial communities. Hybridization was performed in the dark, for $1 \mathrm{~h}$ at $65^{\circ} \mathrm{C}$, as determined elsewhere (Lopes et al., 2017). For washing $\left(65^{\circ} \mathrm{C}\right.$ for $30 \mathrm{~min}$ ), a fresh solution composed of $5 \mathrm{mM}$ Tris Base, $15 \mathrm{mM} \mathrm{NaCl}$ and $1 \%(\mathrm{v} / \mathrm{v}$ ) Triton X-100 (all from Sigma) was prepared less than $24 \mathrm{~h}$ before use. Finally, the chamber slides were allowed to air dry in the dark. An additional staining step with DAPI was performed at the end of the hybridization procedure, covering the surfaces with $20 \mu \mathrm{L}$ of DAPI $(40 \mu \mathrm{g} / \mathrm{mL})$ for $5 \mathrm{~min}$ at room temperature in the dark. Then, immediate observation was elaborated in the fluorescence microscope. Negative controls were performed for each experiment, with no probes added to the hybridization solution. For microscopic visualization, a fluorescence microscope (Olympus BX51, Perafita, Portugal) equipped with the filters sensitive to DAPI (BP 365-370, FT 400, LP 421) and to the signaling molecule of the PNA probes (BP 470-490, FT 500, LP 516 and BP 530-550, FT 570, LP 591, for Alexa 488 and 594, respectively) was used.

\subsection{Statistical analysis}

Data were analyzed using the Prism software package (GraphPad Software version 6.0 for Macintosh). Results were compared by oneway analysis of variance (ANOVA) and applying the Tukey multicomparison post-test. Differences were considered statistically significant at $P$ values $<0.05$.

\section{Results}

\subsection{Biofilm mass and metabolic activity under different oxygen conditions}

In order to inspect for changes in biofilm phenotype under variable oxygen conditions, dual- and three-species biofilms encompassing the CF-associated species I. limosus, D. pigrum and $P$. aeruginosa were grown under AER, MAER and ANAER environments, being further analyzed in terms of biomass and metabolic activity (Fig. 1). An incubation period of $6 \mathrm{~h}$ was required for biofilms to achieve the threshold interval $\left(2 \times 10^{5}\right.$ to $\left.2 \times 10^{6} \mathrm{CFU} / \mathrm{cm}^{2}\right)$ as was previously determined by biofilm adhesion kinetics under the selected oxygen conditions (Fig. S2). Regarding biofilm mass (Fig. 1a), dual-species biofilms encompassing $P$. aeruginosa and I. limosus produced the lowest values in comparison with dual-species biofilms of $P$. aeruginos $a$ and $D$. pigrum and the consortia 
(a)

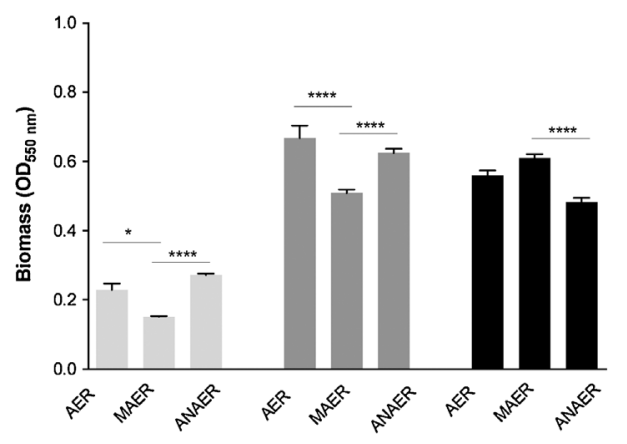

PAIIL (b)

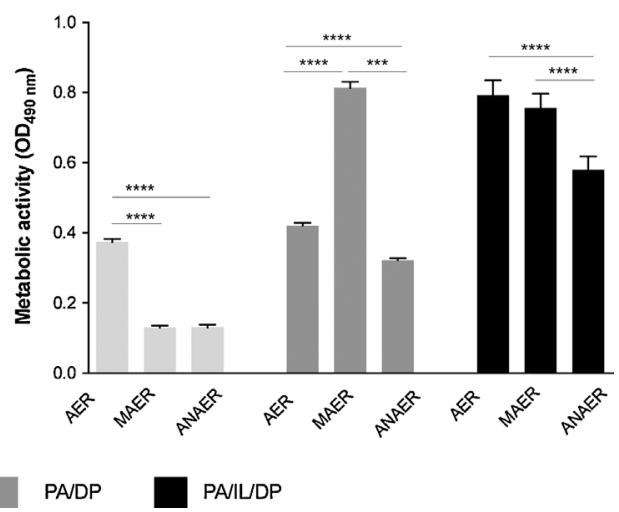

Fig. 1. Characterization of polymicrobial biofilms formed by $P$. aeruginosa (PA), I. limosus (IL) and $D$. pigrum (DP) in terms of (a) biomass and (b) metabolic activity. Biofilms were grown in microtiter plate wells under aerobic (AER), microaerophilic (MAER) and anaerobic (ANAER) atmospheres until achieving the threshold concentration of $2 \times 10^{5}$ to $2 \times 10^{6} \mathrm{CFU} / \mathrm{cm}^{2}(6 \mathrm{~h})$. The means \pm SDs for three independent assays are presented. $* P<0.05$, $* * P<0.01, * * * P<0.001, * * * * P<0.0001$, AER vs. MAER vs. ANAER, one-way ANOVA with Tukey post-hoc test. involving the three species. The same tendency was observed for the metabolic activity of these biofilms (Fig. 1b). Overall, biofilms tend to develop significantly less biomass and present lower metabolic activity for limited oxygen conditions, in particular under ANAER $(P<0.05)$. A particular increase in the metabolic activity was observed for $P$. aeruginosa and $D$. pigrum dual-species consortia developed under a MAER environment.

\subsection{Microbial composition of biofilms under variable oxygen conditions}

In order to investigate which bacterial species were prevailing in the biofilms under AER, MAER and ANAER conditions, the relative population distributions were determined by culture (Fig. 2a). Previously to antibiotic exposure, dual-species biofilms generally presented similar bacterial proportions for all oxygen environments, with $P$. aeruginosa slightly dominating the overall consortia with I. limosus and occupying similar extents with $D$. pigrum. Likewise, the three-species biofilms were clearly dominated by $P$. aeruginos $a$ and $D$. pigrum, whereas I. limosus was the smallest representative population in the overall biofilm. In order to better discriminate and to infer about changes in the distribution and prevalence of the bacterial populations within the multispecies consortia, a multiplex PNA-FISH counterstained with DAPI was employed directly on biofilms (Fig. 2b). Overall, FISH images seemed to qualitatively corroborate culture results. In regard to dual-species biofilms, both populations were detected, with $P$. aeruginosa (red cells) and $D$. pigrum (blue cells) occupying similar extensions in the consortia, but with $P$. aeruginosa slightly dominating the dual-species biofilms encompassing I. limosus. Together with $D$. pigrum, $P$. aeruginosa still prevailed in the three-species consortia, whereas I. limosus populations were sparsely detected under fluorescence microscopy.

\subsection{Interspecies competition within biofilms}

For a clear understanding of the competition between the species (a)

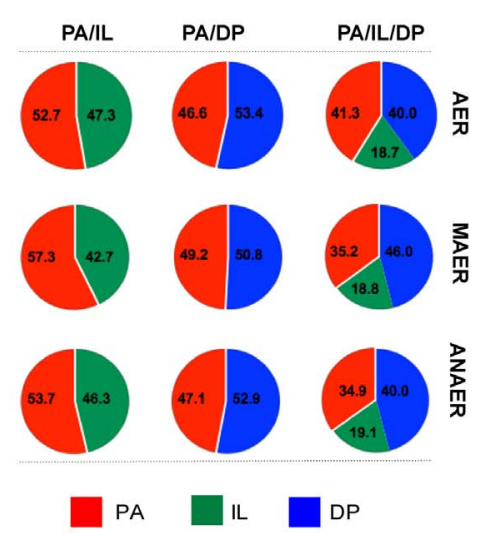

(b)

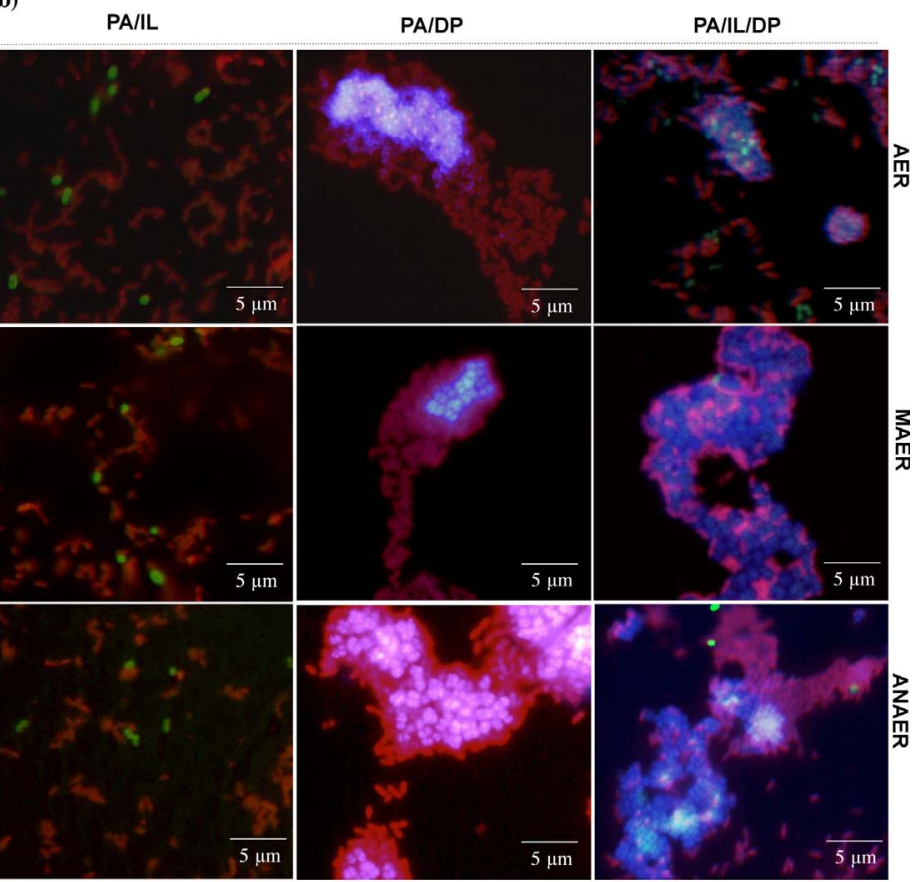

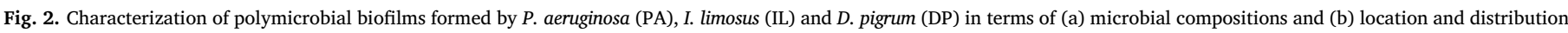

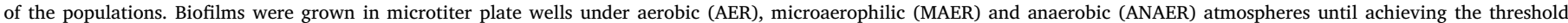

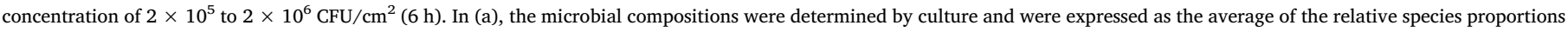

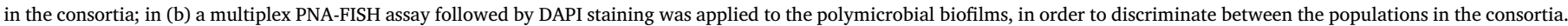

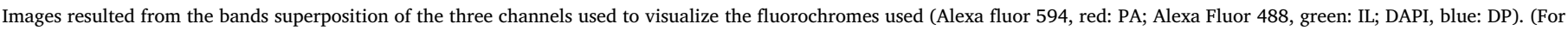
interpretation of the references to colour in this figure legend, the reader is referred to the web version of this article.) 
(a)

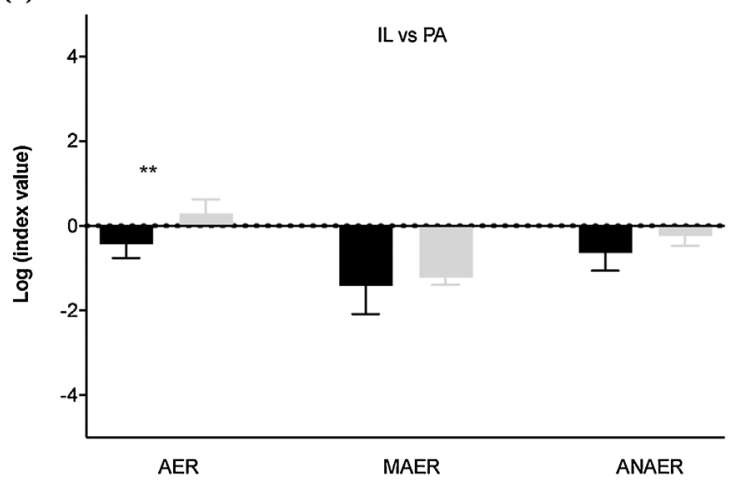

(b)

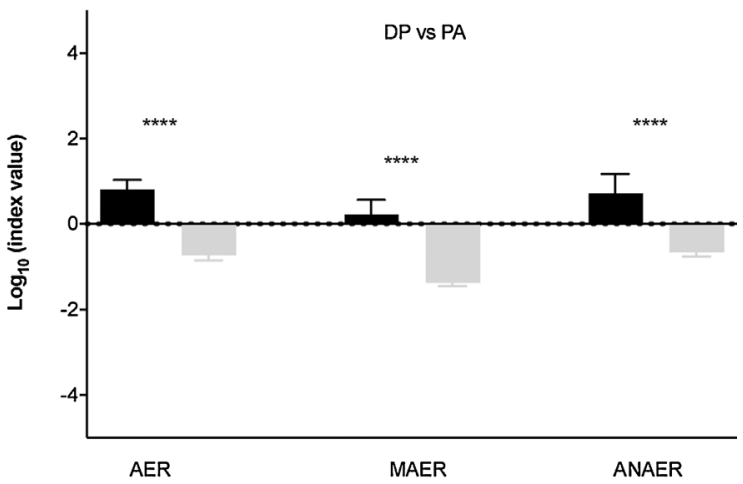

(c)

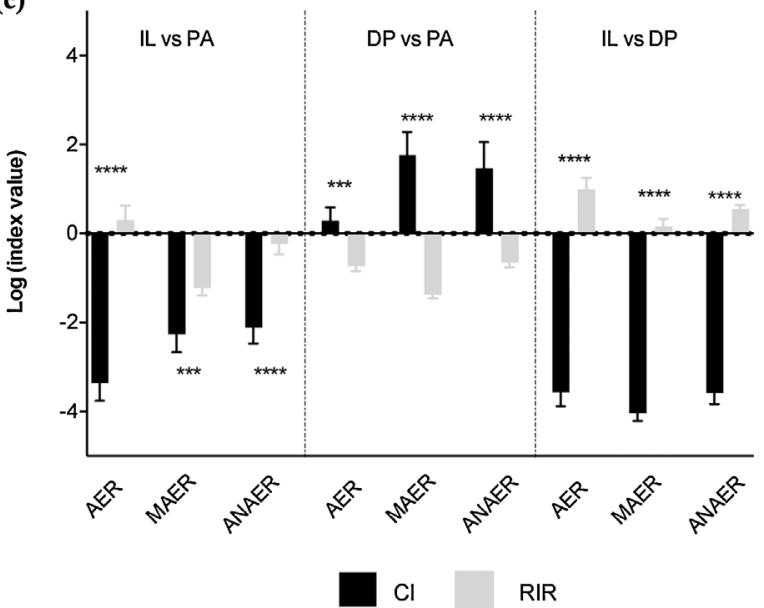

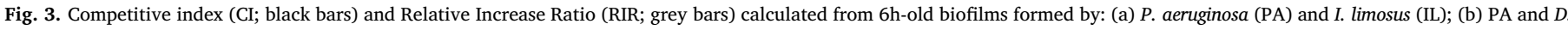

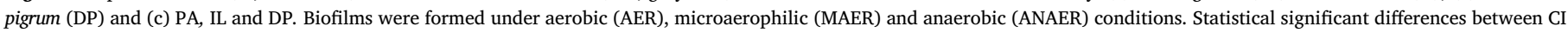

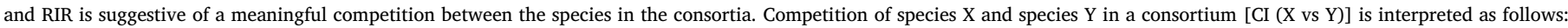

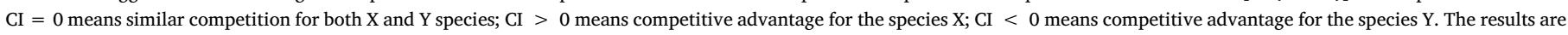
shown as mean \pm SDs. ${ }^{* *} P<0.01,{ }^{* * *} P<0.001,{ }^{* * * * P}<0.0001$, CI vs. RIR, one-way ANOVA with Tukey post-hoc test.

during biofilm growth under AER, MAER and ANAER environments, the CI and RIR indexes were estimated for dual- and three-species biofilms (Fig. 3). Whilst CI allows comparing the differences among the growth of each species in mixed cultures, the RIR index compares the growth of species within pure cultures. As shown, a negative CI index was observed for the $P$. aeruginosa/I. limosus dual-species consortia (Fig. 3a), meaning a competitive advantage for $P$. aeruginosa over $I$. limosus. However, this competition was only significant for AER conditions (CI vs. RIR, $P<0.01$ ). By the contrary, for dual-species consortia involving $P$. aeruginosa/D. pigrum, the unusual species clearly presented competitive advantage over $P$. aeruginosa for all oxygen environments (CI $>0$; CI vs. RIR, $P<0.0001$ ) (Fig. 3b). Similarly, when both unusual species were in co-culture with $P$. aeruginosa (Fig. 3c), I. limosus was outcompeted by $P$. aeruginosa $(\mathrm{CI}<0)$, whereas $D$. pigrum still outcompeted the conventional $\mathrm{CF}$-associated species in the consortia for all oxygen conditions (CI > 0; CI vs. RIR, $P<0.05$ ). Equally, negative $\mathrm{CI}$ indexes indicated $D$. pigrum significantly dominating over $I$. limosus growth within the three-species consortia under all environments (CI $<0$; CI vs. RIR, $P<0.0001$ ). Other approach to represent these latest results consisted in calculating the relative fitness of $P$. aeruginosa when co-cultured with the unusual species (Fig. S3). This demonstrated enhanced $P$. aeruginosa fitness when co-cultured with $I$. limosus and a decline in its performance when D. pigrum is present in the polymicrobial consortia.

\subsection{Biofilm resistance profiles}

The biofilms in the required threshold concentration were exposed to increasing 2-fold concentrations, ranging from 2 to $1024 \mathrm{mg} / \mathrm{L}$, of nine antibiotics. The MICs, obtained from planktonic populations, and the MBECs obtained for biofilm consortia are summarized on Table S1. Results obtained for mixed-species populations were compared to data obtained from single-species cultures, determined in a previous report of Lopes et al. (Lopes et al., 2014), where an increase in antibiotic resistance was observed for I. limosus and D. pigrum biofilms. In order to better visualize the tendency for antibiotic tolerance for those populations, two heat maps representing the MIC and MBEC data were generated (Fig. 4a and b, respectively). Biofilm-entrapped bacteria showed predictable greater tolerance towards antibiotics than planktonic counterparts, with MBECs (in general $\geq 1024 \mathrm{mg} / \mathrm{L}$ ) higher than MICs, independently from the oxygen concentration. For planktonic and biofilm cultures, the activity of most antibiotics was preserved under environments defective in oxygen. Specifically, as aminoglycoside uptake is severely limited in Gram-negative and Gram-positive bacteria under oxygen-restricted environments (Hancock, 1962; Kogut et al., 1965; Kohanski et al., 2010), is therefore indicative that oxygen in the environment (used as the electron acceptor) is the motive force available to assit the aminoglycoside uptake and maintain sensitive under anaerobic conditions (it was the case of $P$. aeruginosa).

Comparing with monospecies planktonic cultures, polymicrobial populations remained sensitive for most antibiotic agents, with exception for CLI and RIF, which were ineffective in inhibiting those 
(a)
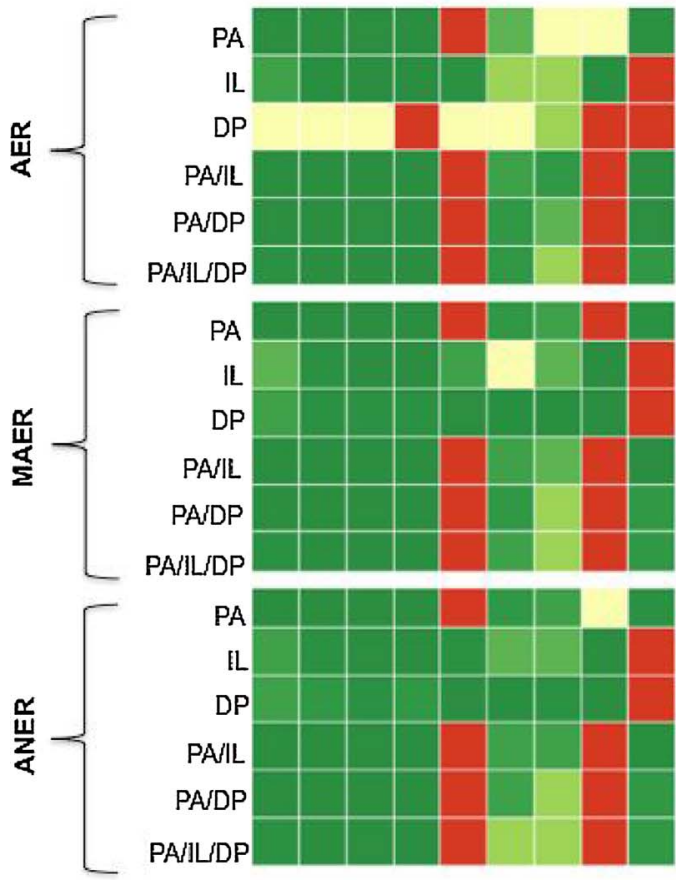

\section{PA}

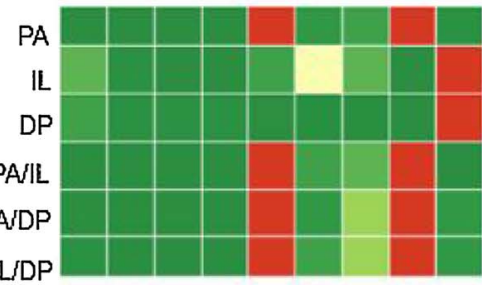

PAILIDP

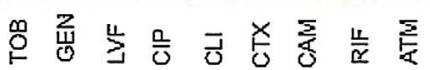

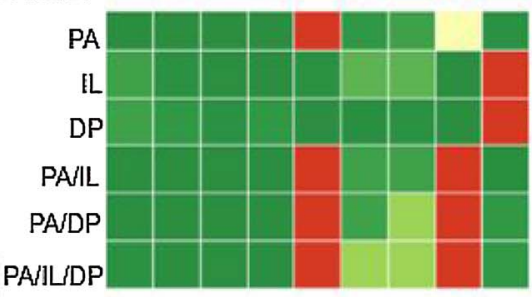

(b)
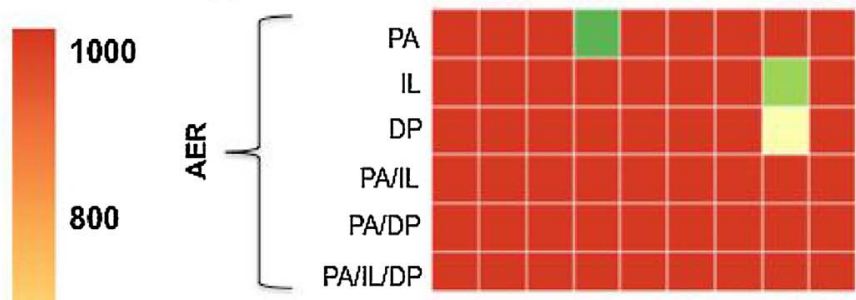

600
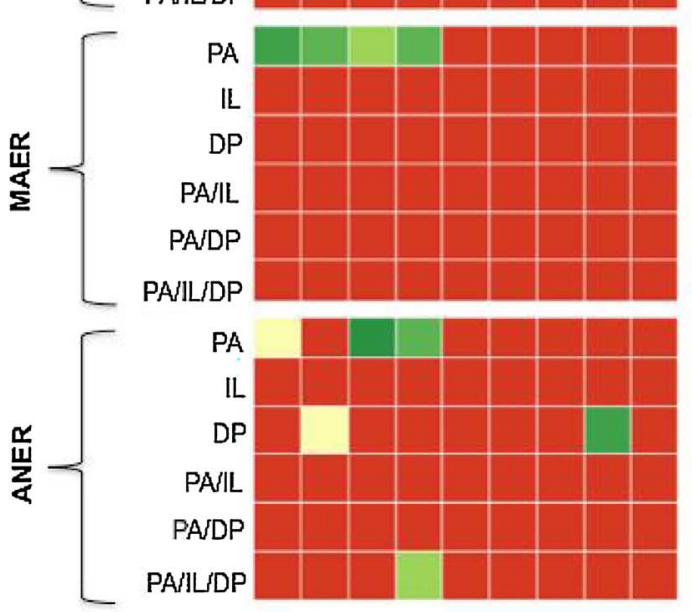

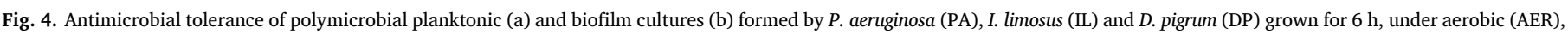

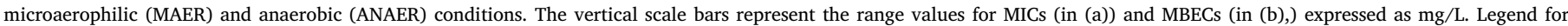

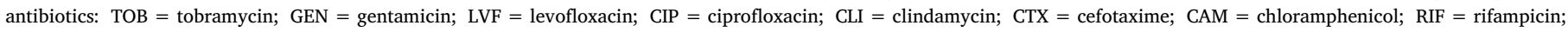

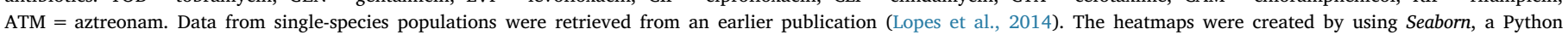
visualization library.

populations (MBEC > $1024 \mathrm{mg} / \mathrm{L}$ ). When encased in biofilms, the unusual species showed high resistance for most antibiotics, which endured when these species were co-cultured with $P$. aeruginosa, independently of the oxygen condition.

\subsection{Effect of antibiotics in reducing biofilm cells}

Since MBECs were generally high, it was interesting to address how increasing antibiotic concentrations (at 2, 128 and $1024 \mathrm{mg} / \mathrm{L}$ ) could affect the viability of bacterial populations within the polymicrobial biofilms (Fig. 5). For this, the percentage reduction in total biofilm-cells was estimated by counting bacteria on TSA after exposing biofilms to antibiotics and the results compared from those of un-treated biofilms. Overall, biofilm-cells declined gradually with increasing doses of antibiotics, with the highest concentrations leading to the highest reductions. A clear loss of activity for many antibiotics under low-oxygen atmospheres was also observed, with no visible cell reductions. This was particularly true for $P$. aeruginosa/I. limosus dual-species biofilms, which showed higher antibiotic resistance under ANAER conditions, with biofilm-cell reduction percentages not exceeding 10\% (achieved for GEN). Biofilms encompassing D. pigrum were more sensitive than the previous ones, being markedly noticeable for the consortia involving the three-species, where antibiotics could achieve great bacterial reductions within the biofilms and even with fluoroquinolones (LVF and CIP) leading to the complete eradication for MAER and ANAER environments.

\subsection{Microbial composition of polymicrobial biofilms after antibiotic treatment}

The relative populations proportions of the polymicrobial biofilms after antibiotic exposure was monitored by culture (Fig. 6). After the antibiotic treatment, substantial changes in the composition of the polymicrobial communities occurred compared with non-treated biofilms (i.e. biofilms pre-exposed to antibiotics), with fluctuations depending on the type and on the concentration of the antibiotic and even on the oxygen conditions. P. aeruginosa/I. limosus dual-species biofilms were still predominately governed by the CF-classical species, particularly under AER conditions (Fig. 6a). However, I. limosus populations started to occupy a significant proportion, together with $P$. aeruginosa, for environments of low oxygen tension (MAER and ANAER). Similarly, $P$. aeruginosa prevailed within the $P$. aeruginos $/ D$. pigrum dual-species consortia (Fig. 6b), persisting alone for many cases. For a vast number of situations, $D$. pigrum was able to persist and inhabit nearly half part of the whole consortia, together with $P$. aeruginosa. Changes in the microbial composition of three-species biofilms showed to be quite complex and variable compared with dual-species consortia (Fig. 6c). A clear dominance of the three-species consortia was typically obtained for $D$. pigrum and/or $P$. aeruginosa, whilst $I$. limosus populations rapidly declining.

\section{Discussion}

The recent explosion of large-scale studies has significantly enhanced our appreciation on the true complexity and diversity of the $\mathrm{CF}$ multispecies communities. Indeed, the airways and lungs of CF patients are often heterogeneous systems enclosing gradients of antibiotics, nutrients, and oxygen (Yang et al., 2011), which have been documented as significant factors driving to the diversification at the community level (Cuthbertson et al., 2016; Goddard et al., 2012; Lee et al., 2014; Quinn et al., 2016; Rogers et al., 2015; van der Gast et al., 2014; van der Gast et al., 2011; Zhao et al., 2012).

Our findings demonstrated that CF polymicrobial communities were not static populations, with biofilm dynamics evolving in response to 
PA/IL
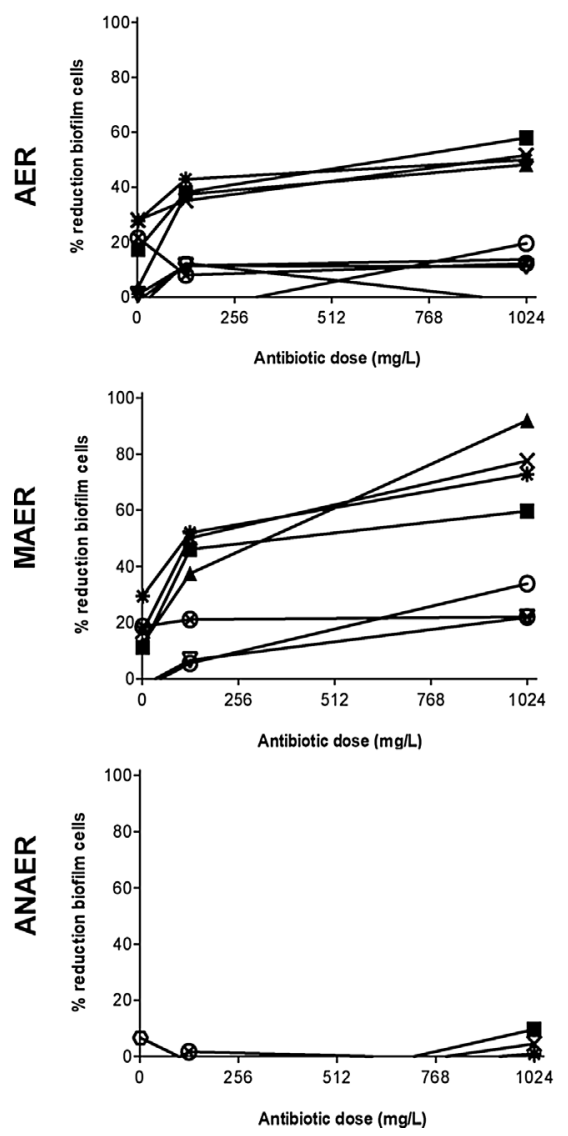

PA/DP
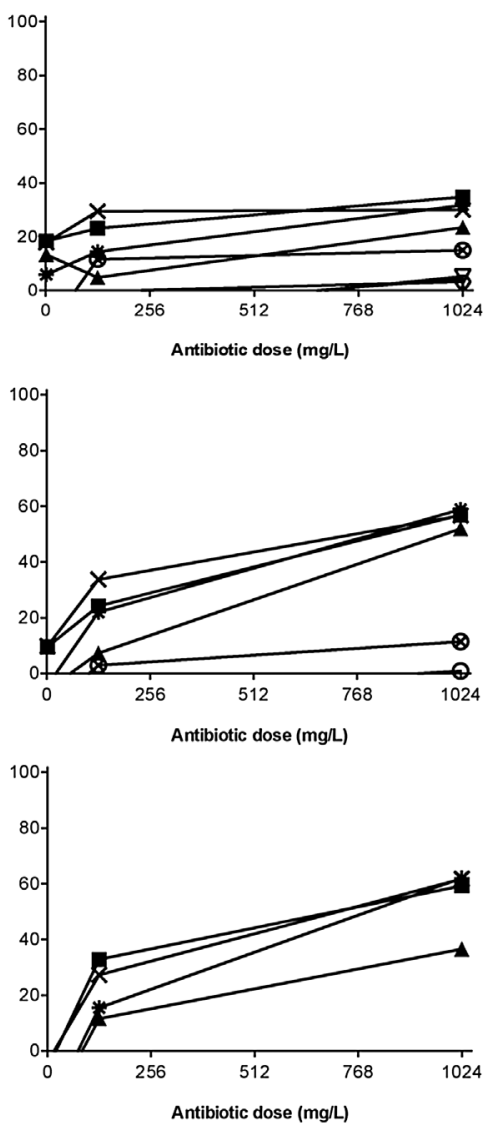

PA/L/DP
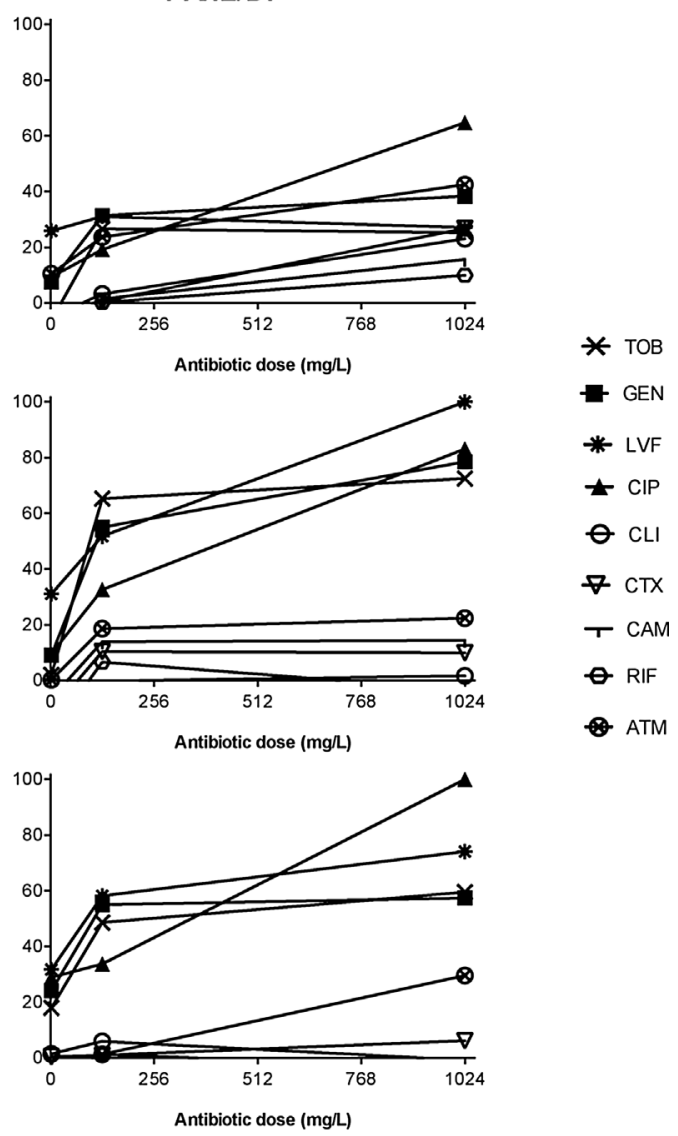

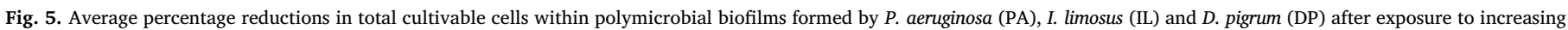

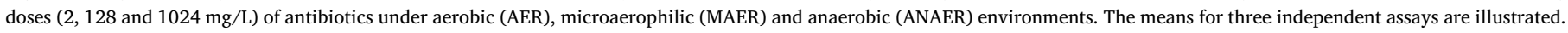

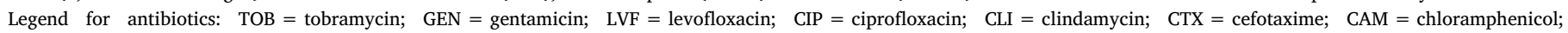
$\mathrm{RIF}=$ rifampicin; ATM $=$ aztreonam.

environments with distinct oxygen availabilities and to antibiotic therapy. Minimal fluctuations were detected before antibiotic exposure, particularly in terms of biofilm composition. In fact, I. limosus and D. pigrum were proficient to establish biofilms together with the CF emblematic pathogen $P$. aeruginosa under AER, MAER and ANAER environments with slight variations in biomass and metabolic activity. Earlier studies (Lopes et al., 2014) have shown that both unusual species were able to develop single-species biofilms under these oxygen environments, even showing resistance to multiple antibiotics under those conditions. Several reasons were pointed out to substantiate the underlying antibiotic resistance for both species, which was particularly attributed to the mucoid physiology of I. limosus and to the high biomass achieved for D. pigrum biofilms, associated to the dense extracellular polymeric matrix.

It is important to note that co-infections with equal ratios of $P$. aeruginosa and the unusual organisms selected may never occur in a CF lung, but it is possible that all strains might reside in the airways of patients for identical lengths in time. Strains of $P$. aeruginosa, I. limosus and $D$. pigrum were recovered from CF exacerbations from one geographical region over a period of just 2 months, which suggests that they may encounter each other in their natural microhabitat (Bittar et al., 2008), eventually not as a stable but as a transient community consequently derived from shifts within the natural environment (Conrad et al., 2013). While applying PNA-FISH assay to discriminate in situ the bacterial populations within the biofilms, culture results were corroborated by microscopy. An exception was observed for $P$. aeruginosa/I. limosus dual biofilms, where the sparse distribution of I. limosus indicated a lower prevalence in the consortium. Only an ex situ quantitative monitoring of bacterial populations should better clarify this issue. Polymicrobial biofilms were minimally challenged by variable oxygen conditions. Whilst dual-species biofilms (in particular $P$. aeruginosa and $D$. pigrum) presented similar relative proportions for both populations, the three-species biofilms were dominated by $P$. aeruginosa, with $D$. pigrum also presenting a great extension together with the CF-traditional species. PNA-FISH and culture assays, complemented with CI and RIR data seems to indicate that D. pigrum/I. limosus and $D$. pigrum/P. aeruginosa interplay was likely negative, with D. pigrum taking competitive advantage against the other species, thus eventually suggesting competition for the same niche or for nutrients (Hibbing et al., 2010).

If biofilm dynamics was minimally disturbed with oxygen conditions, treatment with antibiotics had huge impact on multispecies diversification. First, biofilms demonstrated high resistance towards most antibiotics compared with respective planktonic cells, which is consistent with the commonly accepted notion that biofilm-grown bacteria display enhanced resistance to antibiotics, compared to their planktonic counterparts (Costerton et al., 1999; Hoiby et al., 2010; Lopes et al., 2012; Mah and O'Toole, 2001). This work, thus, supports that the antibiotic resistance previously demonstrated for I. limosus and D. pigrum (Lopes et al., 2014) endured even in co-culture with $P$. aeruginosa, thus strongly emphasizing the impact that those species may have on $\mathrm{CF}$ antibiotic treatment and pathogenesis. Second, even presenting higher MBECs, the antibiotics could lead to significant reductions in the viability of bacterial populations within the polymicrobial biofilms. The decline in the cell viability was less noticed for treatments challenged under ANAER conditions, with a great number of antibiotics losing 
(a)

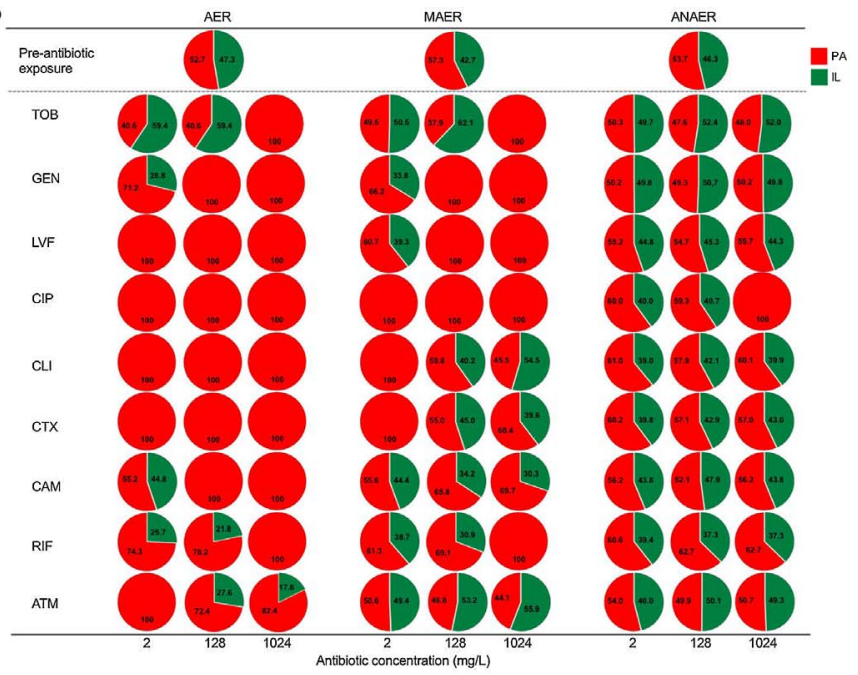

(b)

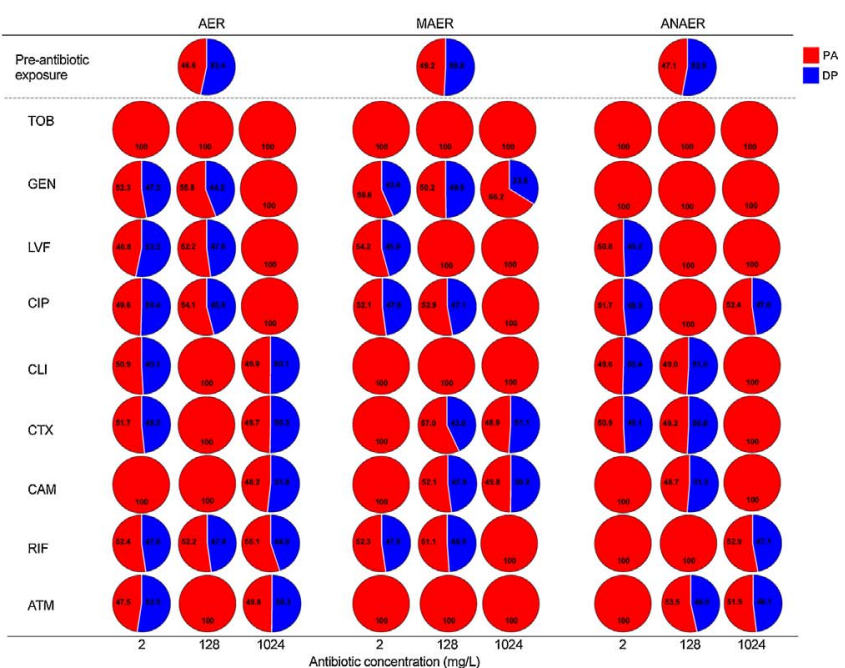

(c)

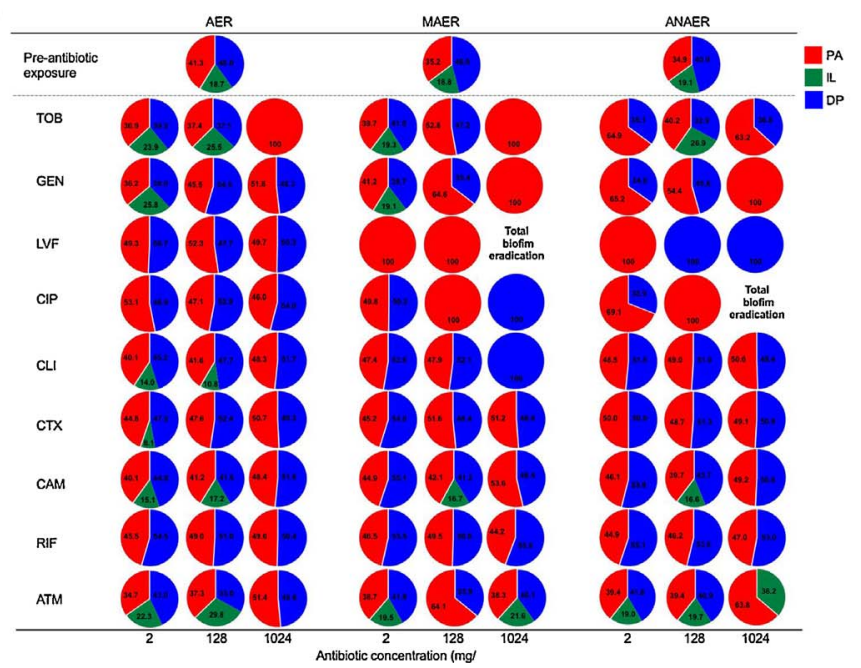

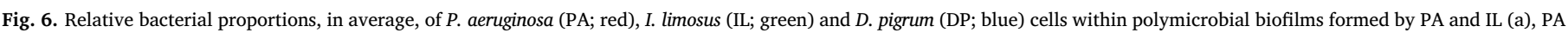

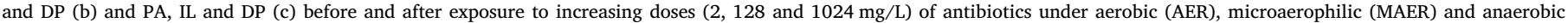

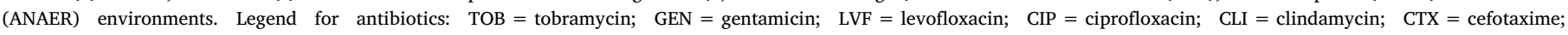

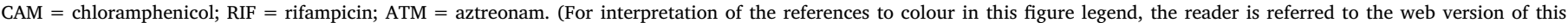
article.)

their effectiveness under oxygen-limited environments. Third, the composition of polymicrobial biofilms was dramatically challenged by antibiotic therapy, with fluctuations depending on the type and concentration of the antibiotic applied and on the oxygen environment. But whatever the antibiotic and concentration applied, it tends to select for $P$. aeruginosa rather than for the unusual species. Changes were in general mainly reflected on the relative proportions of I. limosus and/or $D$. pigrum, with $P$. aeruginosa as the eminent organism for most cases. In turn, changes on biofilms composition instigated by antibiotic therapy could shape the overall function of the polymicrobial consortia. As an example, $P$. aeruginosa/I. limosus dual-species consortia were equally distributed within the biofilm (as showed by culture), mainly under ANAER conditions. The demonstrated endurance of I. limosus populations may indicate that this species was potentially the organism that conferred resistance to the overall biofilm when challenged under low oxygen tensions (observed by bacterial killing assays). We therefore suggest that the enhanced sensitiveness of the three-species consortia can be related with the clear decline in the I. limosus population size, with the overall consortia being dominated by $P$. aeruginos $a$ and $D$. pigrum. This remarks the competition between both unusual species, with D. pigrum likely disturbing the numbers of the organism that originally had conferred resistance to the dual-biofilm (mainly under depleted oxygen conditions) and leading to more susceptible mixed-species biofilms for a great number of antibiotics, as demonstrated by killing kinetics assays. Taken together, these results can open novel ways for CF treatment, by disturbing one factor that regulates the microbial community (in terms of stability, function and/or antibiotic-resistance) to facilitate subsequent control of the overall community by antibiotics.

Aware that rather than a single process, a myriad of variants is responsible for impacting the dynamics of polymicrobial communities in CF natural settings, leading to different scenarios seen in vivo, we were able to reach a model representing how oxygen environments and antibiotic therapy affect the composition of polymicrobial biofilms in CF airways (Fig. 7). For this, we focused our model in the three-species consortia due to their higher complexity comparable with dual-species biofilms. Prior to antibiotic exposure (Fig. 7a), minor fluctuations occur across oxygen gradients, with the three-species consortia being dominated by $P$. aeruginosa (red cells) and $D$. pigrum (blue) and with $I$. limosus (green) as the lowest representative population. When biofilms are exposed to antibiotics commonly used to treat $P$. aeruginosa infections in CF (Doring et al., 2012), diversification among biofilms is evident (Fig. 7b), with variable fluctuations occurring in the relative proportions of the resident species depending on the antibiotic and on the oxygen conditions. 
(a)
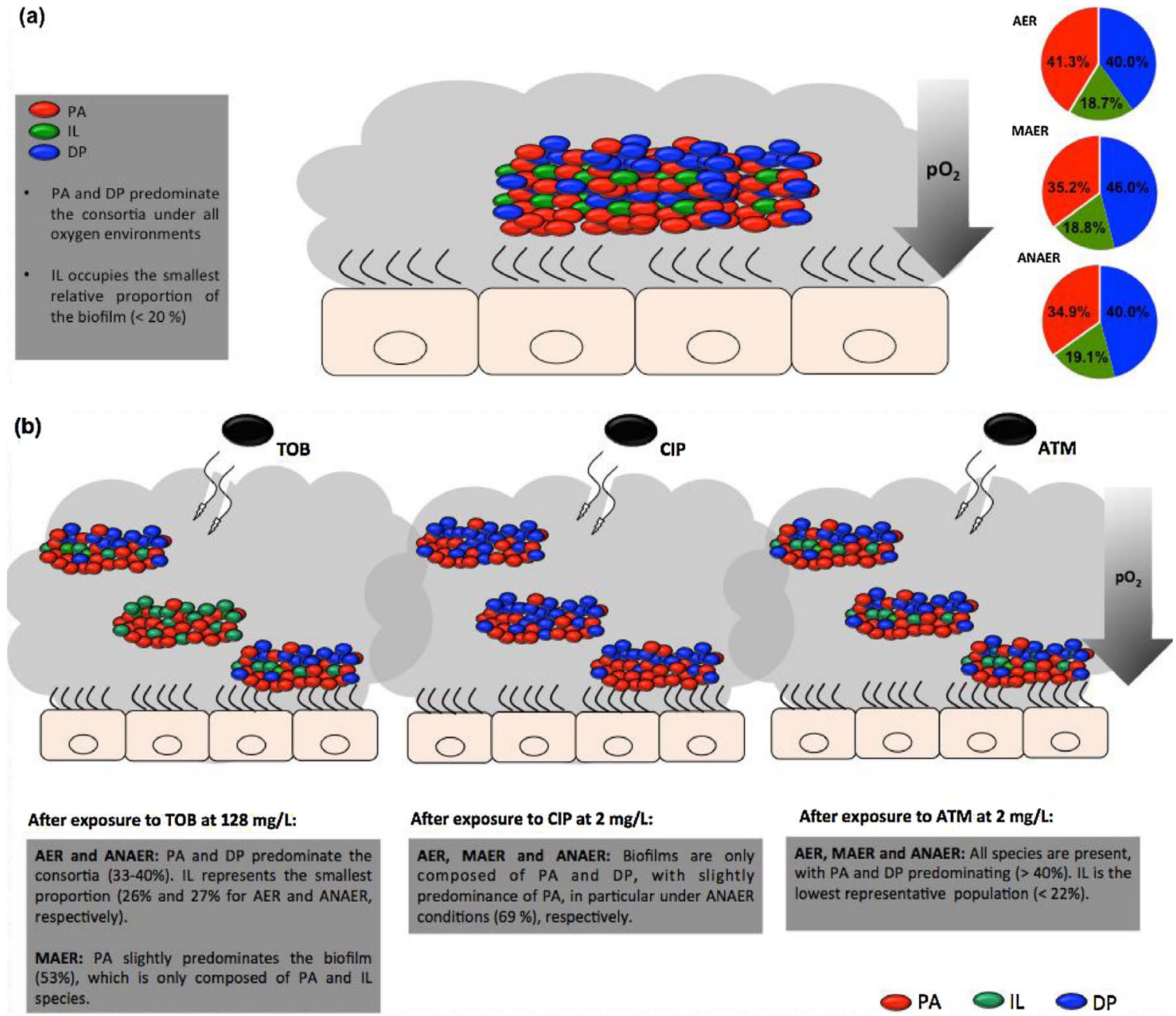

After exposure to CIP at $2 \mathrm{mg} / \mathrm{L}$ :

AER, MAER and ANAER: Biofilms are only composed of PA and DP, with slightly predominance of PA, in particular under ANAER conditions $(69 \%)$, respectively.

\section{After exposure to ATM at $2 \mathrm{mg} / \mathrm{L}$ :}

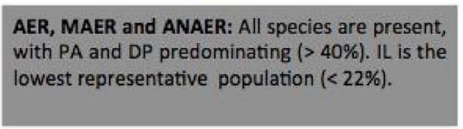

AER, MAER and ANAER: All species are present, with PA and DP predominating ( $>40 \%)$. IL

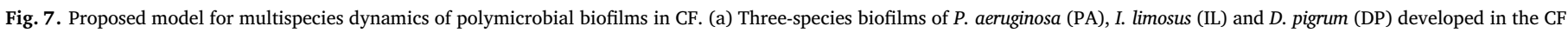

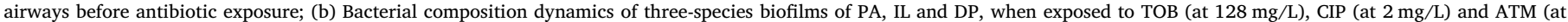
$2 \mathrm{mg} / \mathrm{L}$ ) under aerobiose (AER), microaerophilia (MAER) and anaerobiose (ANAER). Legend: $\mathrm{pO}_{2}=$ oxygen tension; TOB $=$ tobramycin; CIP = ciprofloxacin; ATM = aztreonam.

We are aware that this biofilm model embraces a few limitations (e.g. it is an in vitro model, based on culture experiments complemented with PNA-FISH; it embodies a mixture of three species rather than the full diversity and abundance of microbes in CF and hence dismisses other species with potential huge impact for CF infection). Nonetheless, it reflects how spatially heterogeneous and distributed are CF communities within the site of infection, likely affecting the virulence of the encased pathogens and being a great predictor of disease progression and outcome (Stacy et al., 2016). It is therefore plausible that discerning the mechanisms that govern CF sociomicrobiology will impair, for instance, that treatment regimens often applied to specific populations may be ineffective against infections occurring in particular regions or microenvironments of the lung/airways. Additionally, it may lead to the development of rationales for the selection of suitable and personalized therapeutic strategies (targeting microbiomes unique to each individual), hence increasing antibiotic therapy and reducing socioeconomic repercussion often associated to inadequate treatments.

\section{Conclusion}

This study expanded the understanding about how the dynamics of microbial communities involving $\mathrm{CF}$ classical and unusual organisms is collectively influenced by perturbations often common in the CF natural environments. In fact, our results led to conclude that chemical stress is more "aggressive" than environmental stress in modulating the dynamics of CF polymicrobial communities. While the benefits of antibiotic therapy are indisputable, antibiotics may exert dramatic fluctuations on biofilms, thereby shaping resident species composition and affecting overall function of the consortia. In parallel, this study highlights that the presence of unusual species in the CF airways communities should not be disregarded, since they are able to mediate social interactions, affecting the whole community and ultimately modifying the clinical course of the disease. As such, a more deep appreciation of the ecological and evolutionary nature that shape the airways communities as well as their effects on lung will be certainly useful for the optimal use of current therapies and the development of newer breakthroughs on CF antibiotic therapy.

\section{Funding}

This study was supported by the Portuguese Foundation for Science and Technology (FCT) under the scope of the strategic funding of UID/ BIO/04469/2013 unit and COMPETE 2020 (POCI-01-0145-FEDER006684). The authors also acknowledge the financial support provided by FCT through the projects: PTDC/SAU-ESA/646091/2006/FCOMP01-0124-FEDER-007480FCT; strategic project PEst-OE/EQB/LA0023/ 2013; "BioHealth - Biotechnology and Bioengineering approaches to improve health quality", Ref. NORTE-07-0124-FEDER-000027, cofunded by the Programa Operacional Regional do Norte (ON.2-O Novo Norte), QREN, FEDER; RECI/BBB-EBI/0179/2012 - Consolidating 
Research Expertise and Resources on Cellular and Molecular Biotechnology at CEB/IBB, FCOMP-01-0124-FEDER-027462, FEDER; and the DNA mimics project PIC/IC/82815/2007. The FCT BPD fellowship of Susana P. Lopes SFRH/BPD/95616/2013 and the support of the COST-Action TD1004: Theragnostics for imaging and therapy is also acknowledged.

\section{Conflicts of interest}

None.

\section{Acknowledgements}

The authors would like to thanks to Dr Michael Surette (Farncombe Family Digestive Health Research Institute, McMaster University, Hamilton, ON, Canada) for kindly provide the I. limosus strain and to Fábio Ramalho (Department of Bioinformatics, University of Minho, Braga, Portugal) for creating the heatmaps.

\section{Appendix A. Supplementary data}

Supplementary data associated with this article can be found, in the online version, at http://dx.doi.org/10.1016/j.ijmm.2017.09.018.

\section{References}

Aguirre, M., Morrison, D., Cookson, B.D., Gay, F.W., Collins, M.D., 1993. Phenotypic and phylogenetic characterization of some Gemella-like organisms from human infections: description of Dolosigranulum pigrum gen. nov. sp. nov. J. Appl. Bacteriol. 75, 608-612.

Allen, E.E., Banfield, J.F., 2005. Community genomics in microbial ecology and evolution. Nat. Rev. Microbiol. 3, 489-498.

Azevedo, A.S., Almeida, C., Melo, L.F., Azevedo, N.F., 2014. Interaction between atypical microorganisms and E. coli in catheter-associated urinary tract biofilms. Biofouling 30, 893-902.

Azevedo, A.S., Almeida, C., Pereira, B., Melo, L.F., Azevedo, N.F., 2016. Impact of Delftia tsuruhatensis and Achromobacter xylosoxidans on Escherichia coli dual-species biofilms treated with antibiotic agents. Biofouling 32, 227-241.

Bittar, F., Richet, H., Dubus, J.C., Reynaud-Gaubert, M., Stremler, N., Sarles, J., Raoult, D., Rolain, J.M., 2008. Molecular detection of multiple emerging pathogens in sputa from cystic fibrosis patients. PLoS One 3, e2908.

Bjarnsholt, T., Ciofu, O., Molin, S., Givskov, M., Hoiby, N., 2013. Applying insights from biofilm biology to drug development - can a new approach be developed. Nat. Rev. Drug Discov. 12, 791-808.

Ceri, H., Olson, M.E., Stremick, C., Read, R.R., Morck, D., Buret, A., 1999. The Calgary Biofilm Device: new technology for rapid determination of antibiotic susceptibilities of bacterial biofilms. J. Clin. Microbiol. 37, 1771-1776.

Chiron, R., Marchandin, H., Counil, F., Jumas-Bilak, E., Freydiere, A.M., Bellon, G., Husson, M.O., Turck, D., Bremont, F., Chabanon, G., Segonds, C., 2005. Clinical and microbiological features of Inquilinus sp. isolates from five patients with cystic fibrosis. J. Clin. Microbiol. 43, 3938-3943.

Coenye, T., Goris, J., Spilker, T., Vandamme, P., LiPuma, J.J., 2002. Characterization of unusual bacteria isolated from respiratory secretions of cystic fibrosis patients and description of Inquilinus limosus gen. nov., sp. nov. J. Clin. Microbiol. 40, 2062-2069.

Conrad, D., Haynes, M., Salamon, P., Rainey, P.B., Youle, M., Rohwer, F., 2013. Cystic fibrosis therapy: a community ecology perspective. Am. J. Respir. Cell Mol. Biol. 48, 150-156.

Costerton, J.W., Stewart, P.S., Greenberg, E.P., 1999. Bacterial biofilms: a common cause of persistent infections. Science 284, 1318-1322.

Cuthbertson, L., Rogers, G.B., Walker, A.W., Oliver, A., Green, L.E., Daniels, T.W., Carroll, M.P., Parkhill, J., Bruce, K.D., van der Gast, C.J., 2016. Respiratory microbiota resistance and resilience to pulmonary exacerbation and subsequent antimicrobial intervention. ISME J. 10, 1081-1091.

Davies, J.C., Ebdon, A.M., Orchard, C., 2014. Recent advances in the management of cystic fibrosis. Arch. Dis. Child. 99, 1033-1036.

Doring, G., Flume, P., Heijerman, H., Elborn, J.S., Consensus Study, G., 2012. Treatment of lung infection in patients with cystic fibrosis: current and future strategies. J. Cyst. Fibros. 11, 461-479.

Goddard, A.F., Staudinger, B.J., Dowd, S.E., Joshi-Datar, A., Wolcott, R.D., Aitken, M.L., Fligner, C.L., Singh, P.K., 2012. Direct sampling of cystic fibrosis lungs indicates that DNA-based analyses of upper-airway specimens can misrepresent lung microbiota. Proc. Natl. Acad. Sci. U. S. A. 109, 13769-13774.

Guss, A.M., Roeselers, G., Newton, I.L., Young, C.R., Klepac-Ceraj, V., Lory, S. Cavanaugh, C.M., 2011. Phylogenetic and metabolic diversity of bacteria associated with cystic fibrosis. ISME J. 5, 20-29.

Hancock, R., 1962. Uptake of 14C-streptomycin by some microorganisms and its relation to their streptomycin sensitivity. J. Gen. Microbiol. 28, 493-501.
Hibbing, M.E., Fuqua, C., Parsek, M.R., Peterson, S.B., 2010. Bacterial competition: surviving and thriving in the microbial jungle. Nat. Rev. Microbiol. 8, 15-25.

Hoedemaekers, A., Schulin, T., Tonk, B., Melchers, W.J., Sturm, P.D., 2006. Ventilatorassociated pneumonia caused by Dolosigranulum pigrum. J. Clin. Microbiol. 44, 3461-3462.

Hoiby, N., Bjarnsholt, T., Givskov, M., Molin, S., Ciofu, O., 2010. Antibiotic resistance of bacterial biofilms. Int. J. Antimicrob. Agents 35, 322-332.

Klepac-Ceraj, V., Lemon, K.P., Martin, T.R., Allgaier, M., Kembel, S.W., Knapp, A.A., Lory, S., Brodie, E.L., Lynch, S.V., Bohannan, B.J., Green, J.L., Maurer, B.A., Kolter, R., 2010. Relationship between cystic fibrosis respiratory tract bacterial communities and age genotype, antibiotics and Pseudomonas aeruginosa. Environ. Microbiol. 12, 1293-1303.

Kogut, M., Lightbrown, J.W., Isaacson, P., 1965. Streptomycin action and anaerobiosis. J. Gen. Microbiol. 39, 155-164.

Kohanski, M.A., Dwyer, D.J., Collins, J.J., 2010. How antibiotics kill bacteria: from targets to networks. Nat. Rev. Microbiol. 8, 423-435.

Laclaire, L., Facklam, R., 2000. Antimicrobial susceptibility and clinical sources of Dolosigranulum pigrum cultures. Antimicrob. Agents Chemother. 44, 2001-2003.

Lecuyer, H., Audibert, J., Bobigny, A., Eckert, C., Janniere-Nartey, C., Buu-Hoi, A., Mainardi, J.L., Podglajen, I., 2007. Dolosigranulum pigrum causing nosocomial pneumonia and septicemia. J. Clin. Microbiol. 45, 3474-3475.

Lee, K.W., Periasamy, S., Mukherjee, M., Xie, C., Kjelleberg, S., Rice, S.A., 2014. Biofilm development and enhanced stress resistance of a model, mixed-species community biofilm. ISME J. 8, 894-907.

LiPuma, J.J., 2014. Expanding our understanding of respiratory microbiota in cystic fibrosis. Ann. Am. Thorac. Soc. 11, 1084-1085.

Lopes, S.P., Ceri, H., Azevedo, N.F., Pereira, M.O., 2012. Antibiotic resistance of mixed biofilms in cystic fibrosis: impact of emerging microorganisms on treatment of infection. Int. J. Antimicrob. Agents 40, 260-263.

Lopes, S.P., Azevedo, N.F., Pereira, M.O., 2014. Emergent bacteria in CF-in vitro biofilm formation and resilience under variable oxygen conditions. BioMed Res. Int. 2014 (678307 pages).

Lopes, S.P., Azevedo, N.F., Pereira, M.O., 2015. Microbiome in cystic fibrosis -shaping polymicrobial interactions for advances in antibiotic therapy. Crit. Rev. Microbiol. $41,353-365$.

Lopes, S.P., Carvalho, D.T., Pereira, M.O., Azevedo, N.F., 2017. Discriminating between typical and atypical cystic fibrosis-related bacterial species by multiplex PNA-FISH. Biotechnol. Bioeng. 114, 355-376.

Macho, A.P., Zumaquero, A., Ortiz-Martin, I., Beuzon, C.R., 2007. Competitive index in mixed infections: a sensitive and accurate assay for the genetic analysis of Pseudomonas syringae-plant interactions. Mol. Plant Pathol. 8, 437-450.

Magalhães, A.P., Azevedo, N.F., Pereira, M.O., Lopes, S.P., 2016. The cystic fibrosis microbiome in an ecological perspective and its impact in antibiotic therapy. Appl. Microbiol. Biotechnol. 100, 1163-1181.

Mah, T.F.C., O'Toole, G.A., 2001. Mechanisms of biofilm resistance to antimicrobial agents. Trends Microbiol. 9, 34-39.

Nadell, C.D., Drescher, K., Foster, K.R., 2016. Spatial structure, cooperation and competition in biofilms. Nat. Rev. Microbiol. 14, 589-600.

Pederiva, M.A., Wissmann, G., Friaza, V., Morilla, R., de La Horra, C., Montes-Cano, M.A., Goldani, L.Z., Calderon, E.J., Prolla, J.C., 2012. High prevalence of Pneumocystis jirovecii colonization in Brazilian cystic fibrosis patients. Med. Mycol. 50, 556-560.

Peters, B.M., Jabra-Rizk, M.A., O'May, G.A., Costerton, J.W., Shirtliff, M.E., 2012a. Polymicrobial interactions: impact on pathogenesis and human disease. Clin. Microbiol. Rev. 25, 193-213.

Peters, B.M., Jabra-Rizk, M.A., O'May, G.A., Costerton, J.W., Shirtliff, M.E., 2012b. Polymicrobial interactions: impact on pathogenesis and human disease. Clin. Microbiol. Rev. 25, 193-213.

Quinn, R.A., Phelan, V.V., Whiteson, K.L., Garg, N., Bailey, B.A., Lim, Y.W., Conrad, D.J., Dorrestein, P.C., Rohwer, F.L., 2016. Microbial, host and xenobiotic diversity in the cystic fibrosis sputum metabolome. ISME J. 10, 1483-1498.

Rogers, G.B., van der Gast, C.J., Serisier, D.J., 2015. Predominant pathogen competition and core microbiota divergence in chronic airway infection. ISME J. 9, 217-225.

Schloter, M., Lebuhn, M., Heulin, T., Hartmann, A., 2000. Ecology and evolution of bacterial microdiversity. FEMS Microbiol. Rev. 24, 647-660.

Serisier, D.J., 2013. Risks of population antimicrobial resistance associated with chronic macrolide use for inflammatory airway diseases. Lancet Respir. Med. 1, 262-274.

Stacy, A., McNally, L., Darch, S.E., Brown, S.P., Whiteley, M., 2016. The biogeography of polymicrobial infection. Nat. Rev. Microbiol. 14, 93-105.

Stepanovic, S., Vukovic, D., Dakic, I., Savic, B., Svabic-Vlahovic, M., 2000. A modified microtiter-plate test for quantification of staphylococcal biofilm formation. J. Microbiol. Methods 40, 175-179.

Stevens, M.G., Olsen, S.C., 1993. Comparative-analysis of using Mtt and Xtt in colorimetric assays for quantitating bovine neutrophil bactericidal activity. J. Immunol. Methods 157, 225-231.

Stressmann, F.A., Rogers, G.B., Klem, E.R., Lilley, A.K., Donaldson, S.H., Daniels, T.W., Carroll, M.P., Patel, N., Forbes, B., Boucher, R.C., Wolfgang, M.C., Bruce, K.D., 2011. Analysis of the bacterial communities present in lungs of patients with cystic fibrosis from American and British centers. J. Clin. Microbiol. 49, 281-291.

Tolker-Nielsen, T., Molin, S., 2000. Spatial organization of microbial biofilm communities. Microb. Ecol. 40, 75-84.

van der Gast, C.J., Walker, A.W., Stressmann, F.A., Rogers, G.B., Scott, P., Daniels, T.W., Carroll, M.P., Parkhill, J., Bruce, K.D., 2011. Partitioning core and satellite taxa from within cystic fibrosis lung bacterial communities. ISME J. 5, 780-791.

van der Gast, C.J., Cuthbertson, L., Rogers, G.B., Pope, C., Marsh, R.L., Redding, G.J., Bruce, K.D., Chang, A.B., Hoffman, L.R., 2014. Three clinically distinct chronic pediatric airway infections share a common core microbiota. Ann. Am. Thorac Soc. 11, 
$1039-1048$.

WHO, W.H.O., 2013. Global surveillance, prevention and control of chronic respiratory diseases: a comprehensive approach, Geneva.

Waters, V., 2012. New treatments for emerging cystic fibrosis pathogens other than Pseudomonas. Curr. Pharm. Des. 18, 696-725.

Willner, D., Haynes, M.R., Furlan, M., Schmieder, R., Lim, Y.W., Rainey, P.B., Rohwer, F., Conrad, D., 2012. Spatial distribution of microbial communities in the cystic fibrosis lung. ISME J. 6, 471-474.

Yang, L., Jelsbak, L., Molin, S., 2011. Microbial ecology and adaptation in cystic fibrosis airways. Environ. Microbiol. 13, 1682-1689.

Zhao, J.C., Schloss, P.D., Kalikin, L.M., Carmody, L.A., Foster, B.K., Petrosino, J.F. Cavalcoli, J.D., VanDevanter, D.R., Murray, S., Li, J.Z., Young, V.B., LiPuma, J.J., 2012. Decade-long bacterial community dynamics in cystic fibrosis airways. Proc. Natl. Acad. Sci. U. S. A. 109, 5809-5814. 